\title{
Three categories of set-valued generalisations from fuzzy sets to interval-valued and Atanassov intuitionistic fuzzy sets
}

\author{
Inés Couso and Humberto Bustince, Senior member, IEEE
}

\begin{abstract}
Many different notions included in the fuzzy set literature can be expressed in terms of functionals defined over collections of tuples of fuzzy sets. During the last decades, different authors have independently generalised those definitions to more general contexts, like interval-valued fuzzy sets and Atanassov intuitionistic fuzzy sets. These generalised versions can be introduced either through a list of axioms or in a constructive manner. We can divide them into two further categories: setvalued and point-valued generalized functions. Here we deal with constructive set-valued generalisations. We review a long list of functions, sometimes defined in quite different contexts and we show that we can group all of them into three main different categories, each of them satisfying a specific formulation. We respectively call them the set-valued extension, the max-min extension and the max-min-varied extension. We conclude that the set-valued extension admits a disjunctive interpretation, while the max-min extension can be interpreted under an ontic perspective. Finally, the max-min varied extension provides a kind of compromise between both approaches.
\end{abstract}

Index Terms-Interval-valued fuzzy sets, Atanassov intuitionistic fuzzy sets, extensions of fuzzy sets

\section{INTRODUCTION}

During the last decades, the notion of "fuzzy set" (FS) has been generalized in different ways (see [12]). In particular, the notion of interval-valued fuzzy set (IVFS) was independently considered by Grattan-Guiness [21] and Sambuc [29] (see also [14] for the relation between IVFSs and interval type-2 fuzzy sets). From a different semantic perspective, Atanassov [2] introduced later on the concept of Atanassov "intuitionistic fuzzy set" (A-IFS), that was proved to be formally equivalent to the notion of IVFS [3].

Regarding operations, it is well known that Zadeh proposed to use the principle of extension in order to extend type 1 fuzzy notions to type 2 fuzzy ones, and in particular, to the case of IVFSs. After performing a formal analysis, we have observed that many extensions encountered in the IVF- and the A-IF-literatures follow this strategy. This is the case, for instance, of the notions of union, intersection, or complement of IVF ( [29]) and A-IF ( [2]) sets. A similar strategy is also followed by Szmidt and Kacprzyk [32] and later on by Grzegorzewski [22] in order to define the "probability" of A-IF events, again by Grzegorzewski in order to extend the

Inés Couso is with Departamento de Estadística e I.O. y D.M., Universidad de Oviedo, Spain (e-mail: couso@uniovi.es)

Humberto Bustince is with Departamento de Automática y Computación and Institute of Smart Cities, Universidad Pública de Navarra, Spain (e-mail: bustince@unavarra.es) notion of "degree of inclusion" between fuzzy sets ( [23]), or by Szmidt and Kacprzyk and independently by $\mathrm{Wu}$ and Mendel (see [26], [33], [34]) in order to extend the notion of "sigma-count" or "cardinality" of a fuzzy set [24], among many other notions. All those definitions admit a "conjunctive" ( [17]) interpretation of interval-valued memberships where IVFSs are regarded as collections of "feasible" fuzzy sets, representing incomplete information about a single, ill-known one. Under this approach, functions will be extended by assigning, to each tuple of IVF sets, the collection of images of all the feasible tuples of fuzzy sets it represents. This collection represent our incomplete information about the image of the "true" but ill-known tuple of fuzzy sets, with the length of each membership interval measuring this incompleteness or uncertainty [13].

But not all the extended definitions in the IVF and the AIF-literatures follow this strategy. In the works [4]-[7], for instance, an extension of A-IF sets, the so-called Atanassov intuitionistic multidimensional fuzzy sets (A-IMFSs), are introduced and analyzed in depth. In this case, rather than considering families of fuzzy sets over the same referential set, a Cartesian product of possibly different referential sets is considered, and it is proved how in this setting common operations for A-IF sets can be extended to this new setting, taking advantage of the flexibility of such sets. Although this kind of construction does not fall into the scope of the present work, as we are not considering but just one single referential set, it is remarkable that such an analysis leads to an interesting algebraic structure which would deserve a study by itself.

In this paper, we check that, apart from the first group of definitions inspired in Zadeh's extension principle, we may distinguish at least two additional categories of set-valued extensions. All definitions to be included in the same category share similar patterns, even if they come from completely different contexts. We do not consider in this first study extensions such as the one corresponding to A-IMFSs. This categorized overview of the state-of-the art will contribute to clarify the meaning of each of the three families of extended definitions. After completing our reviewing study, we have realized that the choice of the particular extension strategy does not depend on the specific notion to be extended. As a matter of fact, we have observed that sometimes several authors have independently proposed different extensions of the same original fuzzy set-concept, each of them following a different strategy among the aforementioned ones. Our classification of existing definitions into three main categories 
will help practitioners to select the most appropriate strategy in every particular application. Thus, in those cases where there are different extensions of the same particular definition, they will be able to select the most suitable one. Furthermore, this categorization will also help to contextualize any future extended definition, and to provide it with an appropriate interpretation.

\section{PRELIMINARIES}

Let $\mathcal{F}(U)$ denote the family of fuzzy subsets of the universe, $U$. A fuzzy subset of $U, A \in \mathcal{F}(U)$ is a mapping $A: U \rightarrow[0,1]$. For each element $x \in U$, the value $A(x)$ is called the "membership degree" of $x$ to the fuzzy set $A$. An Atanassov intuitionistic fuzzy set (A-IFS) is a mapping $A: U \rightarrow D([0,1])=\{(x, y) \in[0,1]: x+y \leq 1\}$. It is therefore represented by means of a pair of mappings $\mu_{A}: U \rightarrow[0,1]$ and $\nu_{A}: U \rightarrow[0,1]$ respectively called the "degree of membership" and of "non-membership" satisfying the restriction

$$
\mu_{A}(x)+\nu_{A}(x) \leq 1, \forall x \in U .
$$

An interval-valued fuzzy subset (IVFS) of $U$ is a mapping $A=[\underline{A}, \bar{A}]: U \rightarrow \mathbb{I}[0,1]$, where $\mathbb{I}[0,1]$ denotes the family of closed intervals included in the unit interval $[0,1]$. The mappings $\underline{A}: U \rightarrow[0,1]$ and $\bar{A}: U \rightarrow[0,1]$ respectively determine the lower and upper bounds of the corresponding intervals.

From now on $I F S(U)$ and $I V F S(U)$ will respectively denote the collections of A-IFSs and IVFSs of $U$. According to [3], [19], we formally identify any IVFS $A=[\underline{A}, \bar{A}]$ with an A-IFS $\left(\mu_{A}, \nu_{A}\right)$ by means of the formula:

$$
\mu_{A}(x)=\underline{A}(x), \text { and } \nu_{A}(x)=1-\bar{A}(x), \forall x \in U .
$$

In the rest of the paper we will indistinctly speak about A-IFSs or IVFSs. Any IVFS $A=[\underline{A}, \bar{A}]$ is univocally determined by a pair of fuzzy subsets $(\underline{A}, \bar{A})$ satisfying the restriction $\underline{A} \subseteq \bar{A}$ (where $\subseteq$ denotes the usual min-based inclusion between fuzzy sets). Thus, $A=[\underline{A}, \bar{A}]$ can be regarded as an "interval of fuzzy subsets" wrt the lattice $(\mathcal{F}(U), \subseteq)$, i.e.:

$$
[\underline{A}, \bar{A}]=\{X \in \mathcal{F}(U): \underline{A} \subseteq X \subseteq \bar{A}\} .
$$

The notion of interval of fuzzy sets has been already introduced by Nempont et al. in [25] and can be traced back to the idea of Atanassov operators $K_{\alpha}(\alpha \in[0,1]$, see [2], [10]).

\section{SET-VALUED EXTENSIONS}

From a formal point of view, the different notions in the literature both for IVFSs and for A-IFSs can be seen in many cases as extensions of the original notion in the fuzzy setting which are expressed in terms of a mapping $f$ defined over a tuple of families of fuzzy subsets, $\mathcal{F}(U) \times \ldots \times \mathcal{F}(U)$, and whose images are either fuzzy subsets of $U$ ( as for usual operations between fuzzy sets like the union, the intersection or the complement) or numbers ( as for most of the information measures). These two situations, however, can be seen as particular instances of a generic formulation, even if they deal with completely different contexts. In this paper, we will restrict our attention to set-valued extended mappings. Their images will be therefore either subsets of $\mathcal{F}(U)$ or subsets of the real line, $\mathbb{R}$, depending on the nature of $f$. In the forthcoming subsections, we will consider three different categories, and we will highlight their differences.

A. First category: Interval-valued fuzzy sets regarded as intervals of fuzzy sets

As we have pointed out in the Introduction, an IVFS $A=$ $[\underline{A}, \bar{A}]$ can be regarded as an "interval of fuzzy subsets" of the universe, see Equation 1.

With this idea in mind, we will discuss below a natural procedure that allows us to extend the formulation of certain operations or measures originally defined for fuzzy sets to the case of IVFSs. It has been followed by many scholars in order to extend some well known definitions in fuzzy set theory. We will distinguish two cases: the case where we extend fuzzy sets operators, and the case where we extend real-valued mappings. Formally, the two general formulations respectively provided in Definitions 1 and 2 could be encompassed into a more general formula. Notwithstanding, we will study and illustrate both cases separately, for the sake of clarity.

1) Operations between IF and between IVF sets: An operation between fuzzy sets can be expressed by means of a mapping $O: \mathcal{F}(U) \times \stackrel{(n)}{.} \times \mathcal{F}(U) \rightarrow \mathcal{F}(U)$, where usually $n$ is equal to 1 (unary operations, like the complement, the dilation or the concentration) or 2 (binary operations, like the union or the intersection). The most common operations between fuzzy sets have been independently extended to the case of IVFSs by Sambuc [29] and to the case of A-IFSs by Atanassov [2]. According to the existing bijection $\Phi$ between A-IFSs and IVFSs, we can easily observe that both types of extended definitions are formally equivalent to each other. Furthermore, we will show that those extended definitions are particular cases of the same general formula given below:

Definition 1: The set-valued extension of $O: \mathcal{F}(U) \times(n)$. $\times \mathcal{F}(U) \rightarrow \mathcal{F}(U)$ is the mapping $[O]: I V F S(U) \times(n)$. $\times I V F S(U) \rightarrow \wp(\mathcal{F}(U))$ defined as:

$$
\begin{array}{r}
{[O]\left(A_{1}, \ldots, A_{n}\right)=} \\
\left\{O\left(X_{1}, \ldots, X_{n}\right): \underline{A_{i}} \subseteq X_{i} \subseteq \overline{A_{i}}, i=1, \ldots, n\right\}
\end{array}
$$

for all $\left.\left(A_{1}, \ldots, A_{n}\right) \in I V F S(U) \times \stackrel{(n)}{ }\right) \times I V F S(U)$.

Proposition 1 provides sufficient conditions under which the images of $[O]$ have a maximum and a minimum with respect to the inclusion relation.

Proposition 1: If $O$ is monotonically increasing wrt $k$ components $i_{1}, \ldots, i_{k}$ and monotonically decreasing wrt the remaining ones $i_{k+1}, \ldots, i_{n}$ then the images of its set-valued extension have a maximum and a minimum with respect to the partial ordering determined by fuzzy set inclusion. They are calculated as follows:

$$
\min [O]\left(A_{1}, \ldots, A_{n}\right)=O\left(\left(A_{1}\right)_{*}, \ldots,\left(A_{n}\right)_{*}\right)
$$

and

$$
\max [O]\left(A_{1}, \ldots, A_{n}\right)=O\left(\left(A_{1}\right)^{*}, \ldots,\left(A_{n}\right)^{*}\right),
$$


where $\left(\left(A_{1}\right)_{*}, \ldots,\left(A_{n}\right)_{*}\right)$ and $\left(\left(A_{1}\right)^{*}, \ldots,\left(A_{n}\right)^{*}\right)$ are respectively defined as follows:

- $\left(A_{i}\right)_{*}=A_{i}, i=i_{1}, \ldots, i_{k},\left(A_{i}\right)_{*}=\overline{A_{i}}, i=$
$i_{k+1}, \ldots, i_{n}, \overline{A_{i}}, i=i_{1}, \ldots, i_{k},\left(A_{i}\right)^{*}=\underline{A_{i}}, i=$
- $\left(A_{i}\right)^{*}=\overline{ }=$
$i_{k+1}, \ldots, i_{n}$.

Under the above conditions, the minimum and the maximum of $[O]\left(A_{1}, \ldots, A_{n}\right)$ are calculated as functions of the $2 n$ dimensional tuple of fuzzy sets composed by the extremes of the IVF sets $\left(A_{1}, \ldots, A_{n}\right)$. Proposition 2 provides sufficient conditions in order to guarantee that the images of $[O]$ are intervals of fuzzy sets.

Proposition 2: Suppose that $O: \mathcal{F}(U) \times \ldots \times \mathcal{F}(U) \rightarrow$ $\mathcal{F}(U)$ is defined from $s:[0,1] \times \ldots[0,1] \rightarrow[0,1]$ as follows:

$$
O\left(X_{1}, \ldots, X_{n}\right)(x)=s\left(X_{1}(x), \ldots, X_{n}(x)\right), \forall x \in U .
$$

Then:

- If $s$ is increasing (resp. decreasing) on a component, then $O$ is increasing (resp. decreasing) wrt set inclusion on the same component.

- If $s$ is continuous, then for any arbitrary sequence of pairs of elements of $\mathcal{F}(U), A_{i} \subseteq \overline{A_{i}}, i=1, \ldots, n$, the set $\left\{O\left(X_{1}, \ldots, X_{n}\right): A_{i} \subseteq \bar{X}_{i} \subseteq \overline{A_{i}}, i=1, \ldots, n\right\}$ is an interval of fuzzy subsets of $U$.

Proof: The first result is straightforward and the second one follows from the Weierstrass and the Intermediate Value Theorems, since for an arbitrary $x \in U$, the set

$$
\left\{O\left(X_{1}, \ldots, X_{n}\right)(x): \underline{A_{i}} \subseteq X_{i} \subseteq \overline{A_{i}}, i=1, \ldots, n\right\}
$$

coincides with

$$
\left\{s\left(x_{1}, \ldots, x_{n}\right): \underline{A_{i}}(x) \leq x_{i} \leq \overline{A_{i}}(x)\right\} .
$$

This is the image by $s$ of a rectangle of $\mathbb{R}^{n}$ and therefore, according to those theorems, it is an interval of the form:

$$
\left[s\left(\left(A_{1}\right)_{*}(x), \ldots,\left(A_{n}\right)_{*}(x)\right), s\left(\left(A_{1}\right)^{*}(x), \ldots,\left(A_{n}\right)^{*}(x)\right)\right]
$$

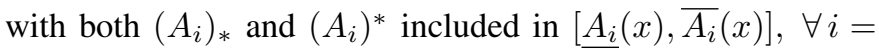
$1, \ldots, n$. Since this happens for any $x \in \bar{U}$, the collection:

$$
\left\{O\left(X_{1}, \ldots, X_{n}\right): \underline{A_{i}} \subseteq X_{i} \subseteq \overline{A_{i}}, i=1, \ldots, n\right\}
$$

coincides with the interval $\left[O\left(\left(A_{1}\right)_{*}, \ldots,\left(A_{n}\right)_{*}\right), O\left(\left(A_{1}\right)^{*}, \ldots,\left(A_{n}\right)^{*}\right)\right]$.

Corollary 1: Suppose that there exists a continuous mapping $s:[0,1] \times \ldots[0,1] \rightarrow[0,1]$ such that $O: \mathcal{F}(U) \times \ldots \times$ $\mathcal{F}(U) \rightarrow \mathcal{F}(U)$ is defined from $s$ as follows:

$$
O\left(X_{1}, \ldots, X_{n}\right)(x)=s\left(X_{1}(x), \ldots, X_{n}(x)\right), \forall x \in U .
$$

Suppose that $s$ is increasing with respect to $k$ components $i_{1}, \ldots, i_{k}$ and monotonically decreasing wrt the remaining ones $i_{k+1}, \ldots, i_{n}$. Let $[O]: \operatorname{IVFS}(U) \times \ldots \times$ $I V F S(U) \rightarrow I V F S(U)$ denote the set-valued extension of $O$. Then $[O]\left(A_{1}, \ldots, A_{n}\right)$ can be expressed as the intervalvalued fuzzy set

$$
\left[O\left(\left(A_{1}\right)_{*}, \ldots,\left(A_{n}\right)_{*}\right), O\left(\left(A_{1}\right)^{*}, \ldots,\left(A_{n}\right)^{*}\right)\right],
$$

$\forall\left(A_{1}, \ldots, A_{n}\right) \in I V F S(U) \times \ldots \times I V F S(U)$, where $\left(\left(A_{1}\right)_{*}, \ldots,\left(A_{n}\right)_{*}\right)$ and $\left(\left(A_{1}\right)^{*}, \ldots,\left(A_{n}\right)^{*}\right)$ are respectively defined as follows:

- $\left(A_{i}\right)_{*}=A_{i}, i=i_{1}, \ldots, i_{k},\left(A_{i}\right)_{*}=\overline{A_{i}}, i=$ $i_{k+1}, \ldots, i_{n}$

- $\left(A_{i}\right)^{*}=\overline{A_{i}}, i=i_{1}, \ldots, i_{k},\left(A_{i}\right)^{*}=\underline{A_{i}}, i=$ $i_{k+1}, \ldots, i_{n}$.

As we have mentioned at the beginning of this section, the most common IVF set operators are particular instances of Definition 1. As an example, let us check that the union of IVF sets defined by Sambuc, and its formally equivalent formulation given by Atanassov follow the general formula provided in Definition 1. The respective proofs for the case of the intersection and the complement are quite similar. The notions of concentration and dilation defined in [27] also follow this general pattern. The notion of cylindric extension defined in [26], [33] is also quite related to this general notion.

Example 1: The union of two IVFSs $A=[\underline{A}, \bar{A}]$ and $B=[\underline{B}, \bar{B}]$ has been defined in [29] as the IVFS $A \cup B=$ $[\underline{A \cup B}, \overline{A \cup B}]$, where:

$$
\underline{A \cup B}=\underline{A} \cup \underline{B} \text {, and } \overline{A \cup B}=\bar{A} \cup \bar{B} .
$$

Now, according to Equation 1, $A \cup B$ can be regarded as the following interval of fuzzy sets:

$$
\{Z \in \mathcal{F}(U): \underline{A} \cup \underline{B} \subseteq Z \subseteq \bar{A} \cup \bar{B}\} .
$$

On the other hand, the "set-valued extension of the union" (Definition 1) is defined as:

$$
\{X \cup Y \in \mathcal{F}(U): \underline{A} \subseteq X \subseteq \bar{A}, \underline{B} \subseteq Y \subseteq \bar{B}\} .
$$

Now, taking into account that the union of fuzzy sets is generated by the max T-conorm, which is a continuous and increasing function (in both components), we deduce from Corollary 1 that the two collections of fuzzy sets of Equations 3 and 4 do coincide. Consequently, the notion of "union" between IVFSs and its formally equivalent A-IF-counterpart follows the general formulation given in Definition 1. An analogous argumentation is valid if more general forms of union (i.e., those based in general t-conorms) are considered.

2) Extensions of real-valued mappings: Definition 1 provided a general formulation for extending an operation between fuzzy sets to the IVFSs setting. A similar kind of extension applies to real-valued mappings defined over collections of tuples of fuzzy sets.

Definition 2: The set-valued extension of $f: \mathcal{F}(U) \times \stackrel{(n)}{(n)}$ $\times \mathcal{F}(U) \rightarrow \mathbb{R}$ is the mapping $[f]: \operatorname{IVFS}(U) \times$ (n). $\times I V F S(U) \rightarrow \wp(\mathbb{R})$ defined as:

$$
[f]\left(A_{1}, \ldots, A_{n}\right)=\left\{f\left(X_{1}, \ldots, X_{n}\right): \underline{A_{i}} \subseteq X_{i} \subseteq \overline{A_{i}}, \forall i\right\},
$$

for all $\left(A_{1}, \ldots, A_{n}\right) \in I V F S(U) \times \stackrel{(n)}{.} \times \operatorname{IVFS}(U)$.

The following result is a consequence of the Intermediate Value Theorem.

Proposition 3: Let $U=\left\{x_{1}, \ldots, x_{m}\right\}$ be a finite set, and suppose that there exist $M: \mathbb{R}^{m} \rightarrow \mathbb{R}$ and $g: \mathbb{R}^{n} \rightarrow \mathbb{R}$, both of them continuous, such that $f\left(X_{1}, \ldots, X_{n}\right)=$ $M\left(g\left(X_{1}\left(x_{1}\right), \ldots, X_{n}\left(x_{1}\right)\right), \ldots, g\left(X_{1}\left(x_{m}\right), \ldots, X_{n}\left(x_{m}\right)\right)\right)$, 
$\forall X_{1}, \ldots, X_{n} \in \mathcal{F}(U)$. Then, the images of its set-valued extension $[f]$ are intervals.

Proof: The arguments of $f$ can be easily identified with elements of $[0,1]^{n \cdot m}$ and therefore, under the above assumptions, $f$ is the composition of two continuous functions and therefore it can be seen as a continuous real-valued mapping defined over $[0,1]^{n \cdot m}$. Furthermore, any tuple $\left(A_{1}, \ldots, A_{n}\right) \in$ $I V F S(U) \times \ldots \times \operatorname{IVFS}(U)$ is identified with a sub-rectangle of $[0,1]^{n \cdot m}$. According to the Intermediate Value Theorem, its image through $f$ is a convex subset of $\mathbb{R}$ (an interval).

Proposition 4 provides sufficient conditions for $[f]$ to be expressed as a function of the $2 n$-dimensional tuple $\left(f\left(\underline{A_{1}}\right), \ldots, f\left(\underline{A_{n}}\right), f\left(\overline{A_{1}}\right), \ldots, f\left(\overline{A_{n}}\right)\right)$ :

Proposition 4: If $f$ is monotonically increasing wrt $k$ components $i_{1}, \ldots, i_{k}$ and monotonically decreasing wrt the remaining ones $i_{k+1}, \ldots, i_{n}$ then the images of its set-valued extension satisfy:

$$
\min [f]\left(A_{1}, \ldots, A_{n}\right)=f\left(\left(A_{1}\right)_{*}, \ldots,\left(A_{n}\right)_{*}\right)
$$

and

$$
\max [f]\left(A_{1}, \ldots, A_{n}\right)=f\left(\left(A_{1}\right)^{*}, \ldots,\left(A_{n}\right)^{*}\right)
$$

and therefore, its convex hull coincides with the interval

$$
\left[f\left(\left(A_{1}\right)_{*}, \ldots,\left(A_{n}\right)_{*}\right), f\left(\left(A_{1}\right)^{*}, \ldots,\left(A_{n}\right)^{*}\right)\right],
$$

where $\left(\left(A_{1}\right)_{*}, \ldots,\left(A_{n}\right)_{*}\right)$ and $\left(\left(A_{1}\right)^{*}, \ldots,\left(A_{n}\right)^{*}\right)$ are respectively defined as follows:

- $\left(A_{i}\right)_{*}=A_{i}, i=i_{1}, \ldots, i_{k},\left(A_{i}\right)_{*}=\overline{A_{i}}, i=$ $i_{k+1}, \ldots, i_{n}$

- $\left(A_{i}\right)^{*}=\overline{A_{i}}, i=i_{1}, \ldots, i_{k},\left(A_{i}\right)^{*}=\underline{A_{i}}, i=$ $i_{k+1}, \ldots, i_{n}$

Furthermore, if $f$ satisfies either the following linearity condition:

$$
\begin{aligned}
& f\left(\lambda\left(A_{1}\right)_{*}+(1-\lambda)\left(A_{1}\right)^{*}, \ldots, \lambda\left(A_{n}\right)_{*}+(1-\lambda)\left(A_{n}\right)^{*}\right) \\
& \left.=\lambda f\left(\left(A_{1}\right)_{*}, \ldots,\left(A_{n}\right)_{*}\right)\right)+(1-\lambda) f\left(\left(A_{1}\right)^{*}, \ldots,\left(A_{n}\right)^{*}\right)
\end{aligned}
$$

or the continuity condition considered in Proposition 3, then $[f]\left(A_{1}, \ldots, A_{n}\right)$ coincides with the interval:

$$
\left[f\left(\left(A_{1}\right)_{*}, \ldots,\left(A_{n}\right)_{*}\right), f\left(\left(A_{1}\right)^{*}, \ldots,\left(A_{n}\right)^{*}\right)\right] .
$$

Proof: The first part is straightforward. Let us now prove the second part. Let us assume that $f$ satisfies the linearity condition considered in Equation 6 . We just need to prove that any $c \in\left[f\left(\left(A_{1}\right)_{*}, \ldots,\left(A_{n}\right)_{*}\right), f\left(\left(A_{1}\right)^{*}, \ldots,\left(A_{n}\right)^{*}\right)\right]$ belongs to $[f]\left(A_{1}, \ldots, A_{n}\right)$. For an arbitrary $c$, there exists $\lambda \in[0,1]$ such that $c=\lambda f\left(\left(A_{1}\right)_{*}, \ldots,\left(A_{n}\right)_{*}\right)+(1-\lambda) f\left(\left(A_{1}\right)^{*}, \ldots,\left(A_{n}\right)^{*}\right.$. Let us now consider the tuple of fuzzy sets $\left(C_{1}, \ldots, C_{n}\right)$, where:

$$
C_{i}(x)=\lambda\left(A_{i}\right)_{*}(x)+(1-\lambda) A_{i}^{*}(x), \forall x \in U, i=1, \ldots, n .
$$

According to the above linearity condition, we have that $f\left(C_{1}, \ldots, C_{n}\right)=c$. Furthermore, we can easily check that $A_{i}=\subseteq\left(A_{i}\right)_{*} \cap\left(A_{i}\right)^{*} \subseteq C \subseteq\left(A_{i}\right)_{*} \cup\left(A_{i}\right)^{*}=$ $\overline{A_{i}}, \overline{i=1}, \ldots, n$. Therefore $f\left(C_{1}, \ldots, C_{n}\right)$ belongs to the set $[f]\left(A_{1}, \ldots, A_{n}\right)$ by definition. The last part is a direct consequence of Proposition 3 and the first part of this proposition (Proposition 4).
From now on, we will denote the extremes of the interval $C H\left([f]\left(A_{1}, \ldots, A_{n}\right)\right)$ respectively by:

$$
\underline{[f]}\left(A_{1}, \ldots, A_{n}\right)=f\left(\left(A_{1}\right)_{*}, \ldots,\left(A_{n}\right)_{*}\right)
$$

and

$$
\overline{[f]}\left(A_{1}, \ldots, A_{n}\right)=f\left(\left(A_{1}\right)^{*}, \ldots,\left(A_{n}\right)^{*}\right) .
$$

Some definitions from the literature like the notion of probability of an A-IF event introduced in [32] and also considered in [22] and [28], the cardinality of A-IFSs ( [8], [33]) and of IVFSs [34], the inclusion indicator ( [23]), or the interval-valued similarities considered in [31] are particular instances of the above formulation, as we will check below. Let us start by recalling the notion of probability of an A-IF event initially introduced by Szmidt and Kacprzyk [32] for finite universe and later on considered by Grzegorzewki [22] and by Riecan in [28]:

Definition 3: Let $(U, \mathcal{A}, P)$ be a probability space. Let $A=$ $\left(\mu_{A}, \nu_{A}\right): U \rightarrow[0,1]^{2}$ be an intuitionistic fuzzy event, i.e., an $\mathcal{A}$-Borel $\left(\mathbb{R}^{2}\right)$ measurable mapping. The probability of $A$ is defined as an element in the interval

$$
P(A)=\left[\int \mu_{A} d P, 1-\int \nu_{A} d P\right] .
$$

The above definition clearly generalizes the notion of "probability of a fuzzy event" given by Zadeh in [37]. In fact, if we consider the pair of fuzzy subsets $\underline{A}=\mu_{A}$ and $\bar{A}=1-\nu_{A}$ associated to the intuitionistic fuzzy event $A$, we clearly observe that the interval-valued probability of $A$ can be expressed as $[\tilde{P}(\underline{A}), \tilde{P}(\bar{A})]$, where $\tilde{P}$ denotes Zadeh's probability, as recalled by the authors. Furthermore, due to the monotonicity of $\tilde{P}$ with respect to fuzzy set inclusion, and according to Proposition 4, it coincides with the convex hull of the set-valued extension,

$C H([\tilde{P}](A))=C H(\{P(X): \underline{A} \subseteq X \subseteq \bar{A}, X$ measurable $\})$.

According to Proposition 4 , and due to the linearity of $\tilde{P}$, we can additionally check that the set-valued extension $[\tilde{P}](A)$ is an interval, and therefore it coincides with its convex hull, i.e.

$$
[\tilde{P}](A)=C H([\tilde{P}](A)) .
$$

Let us now recall the notion of interval-valued cardinality of an IVFS ( [26], [33], [34]):

Definition 4: Let us consider an IVFS of $U, A=[\underline{A}, \bar{A}]$. We define its cardinality as the interval of values $[\#](A)=$ $[\# \underline{A}, \# \bar{A}]$, where $\#: \mathcal{F}(U) \rightarrow \mathbb{R}$ denotes the cardinality of fuzzy sets defined by De Luca and Termini as $\#(X)=$ $\sum_{x \in U} X(x), \forall x \in U$.

This is again a particular instance of Definition 2. In fact, according to Proposition 4, the convex hull of the set-valued extension,

$$
C H([\#](A))=C H(\{\# X: X \in \mathcal{F}(U), \underline{A} \subseteq X \subseteq A\})
$$

coincides with $[\# \underline{A}, \# \bar{A}]$. Furthermore, according to the second part of the same proposition, and taking the linearity of the cardinal into account, we easily observe that such a set-valued extension is convex. 
Let us now recall the extension of the notion of inclusion indicator proposed by Grzegorzewski in [23]. He starts by considering inclusion indicators for fuzzy sets in the sense of the axiomatic definition by Cornelis et al. ( [15]):

Definition 5: An inclusion indicator Inc: $\mathcal{F}(U) \times \mathcal{F}(U) \rightarrow$ $\mathbb{R}$ is a function satisfying the following properties:

A1.- $\operatorname{Inc}(X, Y)=1$ if and only if $X \subseteq Y$ (in Zadeh's sense).

A2.- $\operatorname{Inc}(X, Y)=0$ if and only if there exists $x \in U$ with $X(x)=1$ and $Y(x)=1$.

A3.- $\operatorname{Inc}(X, Y)=\operatorname{Inc}\left(Y^{c}, X^{c}\right)$.

A4.- $\operatorname{Inc}(X, Y \cap Z)=\min \{\operatorname{Inc}(X, Y), \operatorname{Inc}(X, Z)\}$.

A5.- $\operatorname{Inc}(X, Y)=\operatorname{Inc}(S(X), S(Y))$, where $S: \mathcal{F}(U) \rightarrow$ $\mathcal{F}(U)$ is a mapping defined, for every $X \in \mathcal{F}(U)$ as $S(X)(x)=X(s(x)), \forall x \in U$, for a certain bijection $s: U \rightarrow U$.

Observe that, although we have focused here on Sinha and Dougherty' approach to inclusion, some other approaches can be found in the literature, see Bustince [11]. In any case, Cornelis et al. proved that the above collection of the five axioms was equivalent to the collection of nine axioms initially proposed by Sinha and Dougherty [30] in order to quantify the degree of inclusion of a fuzzy set in another fuzzy set. Grzegorzewski generalizes the notion of inclusion indicator and defines the indicators of necessary an of possible inclusion as follows:

Definition 6: Let Inc: $\mathcal{F}(U) \times \mathcal{F}(U) \rightarrow \mathbb{R}$ be an inclusion indicator in the sense of Definition 5. Let $A, B \in I F(U)$ be a pair of A-IFSs of $U$. Let $[\underline{A}, \bar{A}]$ and $[\underline{B}, \bar{B}]$ be their respective interval representations.

The indicator of necessary inclusion of $(A, B)$ is defined as

$$
N I(A, B)=\operatorname{Inc}(\bar{A}, \underline{B})
$$

The indicator of possible inclusion of $(A, B)$ is defined as

$$
P I(A, B)=\operatorname{Inc}(\underline{A}, \bar{B}) .
$$

According to the properties of inclusion indicators with respect to Zadeh's inclusion (they can be proved to be increasing wrt the second component and decreasing wrt the first one), and taking the first result in Proposition 4 into account, we can easily observe that the above necessity and possibility indices satisfy the following equality for any pair of A-IFSs $A, B \in I F S(U)$ :

$$
\begin{array}{r}
{[N I(A, B), P I(A, B)]=} \\
C H(\{I(X, Y): \underline{A} \subseteq X \subseteq \bar{A}, \underline{B} \subseteq Y \subseteq \bar{B}\}),
\end{array}
$$

where $C H$ denotes the convex hull. In other words, $N I(A, B)$ and $P I(A, B)$ respectively coincide with the minimum and the maximum of the set:

$$
\{I(X, Y): \underline{A} \subseteq X \subseteq \bar{A}, \underline{B} \subseteq Y \subseteq \bar{B}\} .
$$

Cornelis et al. in [15] proved that a mapping $\operatorname{Inc}: \mathcal{F}(U) \times$ $\mathcal{F}(U) \rightarrow \mathbb{R}$ satisfies the axioms of Sinha-Dougherty if and only if it can be expressed as follows:

$$
\operatorname{Inc}(A, B)=\min _{x \in U} I(A(x), B(x)),
$$

where $I:[0,1] \times[0,1] \rightarrow \mathbb{R}$ is a contrapositive implicator satisfying in addition:

- $x \leq y \Leftrightarrow I(x, y)=1$

- $x=1, y=0 \Leftrightarrow I(x, y)=0$.

If furthermore $I$ is continuous on $[0,1] \times[0,1]$ then we can prove, in virtue of Proposition 4, that the set

$$
\{I(X, Y): \underline{A} \subseteq X \subseteq \bar{A}, \underline{B} \subseteq Y \subseteq \bar{B}\}
$$

is convex and therefore it coincides with the interval $[N I(A, B), P I(A, B)]$. Thus, under these restrictions, Grzegorzewski's notion is a particular instance of the general formulation established in Definition 2.

Let us finally recall the notion of interval-valued similarity considered by A. Stachowiak and K. Dyczkowski in [31] (see [9] and [1] for alternative proposals on interval-valued similarities):

Definition 7: Let $S: \mathcal{F}(U) \times \mathcal{F}(U) \rightarrow \mathbb{R}$ be a similarity measure. Given two IVFSs $A=[\underline{A}, \bar{A}]$ and $B=[\underline{B}, \bar{B}]$, the interval-valued similarity between $A$ and $B$ is defined as:

$$
\begin{gathered}
{[[S]](A, B)=[\underline{[S]}(A, B), \overline{[S]}(A, B)] \text { with }} \\
\underline{[S]}(A, B)=\min _{X \in[\underline{A}, \bar{A}], Y \in[\underline{B}, \bar{B}]} S(X, Y), \\
\left.\overline{[S]}(A, B)=\max _{X \in[\underline{A}, \bar{A}], Y \in[\underline{B}, \bar{B}]} S(X, Y)\right] .
\end{gathered}
$$

The images of $[[S]]$ coincide with the convex hulls of the images of the set-valued extension $[S]$. The authors provide an exact calculation of the upper bound of $[[S]](A, B)$ in terms of two fuzzy sets $S(X, Y)$ whose respective membership values $X(x)$ and $Y(x)$ belong to the collection of extreme membership values $\{\underline{A}(x), \bar{A}(x), \underline{B}(x), \bar{B}(x)\}$ for every $x \in$ $U$. They also propose an approximate computation of the lower bound.

We may conclude from this section that many extensions of FS definitions consider IVFSs as intervals of "feasible" fuzzy sets. Then, the image through $[O]$ (resp. through $[f]$ ) of any tuple, $\left(\left[A_{1}, \overline{A_{1}}\right], \times,\left[\underline{A_{n}}, \overline{A_{n}}\right]\right) \in I V F(U) \times \ldots \times I V F(U)$ is defined as the collection of possible images $O\left(X_{1}, \ldots, X_{n}\right)$ (resp. $f\left(X_{1}, \ldots, X_{n}\right)$ ), when $\left(X_{1}, \ldots, X_{n}\right)$ ranges over the collection of "feasible tuples" of fuzzy subsets of $U$. Under appropriate monotonicity restrictions, of the maximum and the minimum of the extended images can be easily calculated. The next section continues this exploration.

\section{B. Second category: Definitions based on pairs of evaluations over the extremes of the IVF sets}

The procedure reviewed in the last section considered any tuple of IVFSs as a collection of "feasible" tuples of fuzzy sets. The image via $[O]$ or $[f]$ of such a collection of tuples was naturally defined as the set-valued image containing the images of all those feasible tuples. This seems the most natural extension procedure when IVFSs are used in order to represent incomplete knowledge about a specific fuzzy set. The image via $[f]$ or $[O]$ represents the collection of possible images, according to the available partial information. Another reasonable procedure, based on the pair of evaluations of the 
function over the lower and upper extremes, has been also considered. We will provide new general formulations for this alternative procedure and analyze the connections with the previous formulations proposed in Definitions 1 and 2. We will again distinguish between operations between IVFSs and real-valued mappings, for the sake of clarity.

1) Operations between A-IFS and between IVFS: Let us provide a general formulation in order to extend an operator $O: \mathcal{F}(U) \times \stackrel{(n)}{n} \times \mathcal{F}(U) \rightarrow \mathcal{F}(U)$ to a family of tuples of IVFSs. It will be based on the evaluation of $O$ over the extremes of the IVFSs.

Definition 8: The max-min extension of $O: \mathcal{F}(U) \times \stackrel{(n)}{\text {. }}$ $\times \mathcal{F}(U) \rightarrow \mathcal{F}(U)$ is the mapping $\underline{\bar{O}}=[\underline{O}, \bar{O}]$ : $I V F S(U) \times \stackrel{(n)}{ } \ln \times \operatorname{IVFS}(U) \rightarrow I V F S(U)$ defined as follows:

$$
\begin{array}{r}
\underline{O}\left(A_{1}, \ldots, A_{n}\right)(x)= \\
\min \left\{O\left(\underline{A_{1}}, \ldots, \underline{A_{n}}\right)(x), O\left(\overline{A_{1}}, \ldots, \overline{A_{n}}\right)(x)\right\} . \\
\bar{O}\left(A_{1}, \ldots, A_{n}\right)(x)= \\
\max \left\{O\left(\underline{A_{1}}, \ldots, \underline{A_{n}}\right)(x), O\left(\overline{A_{1}}, \ldots, \overline{A_{n}}\right)(x)\right\} .
\end{array}
$$

According to Definition 8, the fuzzy set $\underline{O}\left(A_{1}, \ldots, A_{n}\right)$ (resp. the fuzzy set $\left.\bar{O}\left(A_{1}, \ldots, A_{n}\right)\right)$ does not necessarily coincide either with $O\left(A_{1}, \ldots, A_{n}\right)$ or with $O\left(\overline{A_{1}}, \ldots, \overline{A_{n}}\right)$. In fact, there may exist a couple of elements $x \neq x^{\prime}$ such that

$$
O\left(\underline{A_{1}}, \ldots, \underline{A_{n}}\right)(x)<O\left(\overline{A_{1}}, \ldots, \overline{A_{n}}\right)(x)
$$

and

$$
O\left(\underline{A_{1}}, \ldots, \underline{A_{n}}\right)\left(x^{\prime}\right)<O\left(\overline{A_{1}}, \ldots, \overline{A_{n}}\right)\left(x^{\prime}\right),
$$

and therefore $\underline{O}\left(A_{1}, \ldots, A_{n}\right)$ is strictly included in both fuzzy sets. The following proposition establishes sufficient conditions under which the fuzzy sets $O\left(\underline{A_{1}}, \ldots, \underline{A_{n}}\right)$ and $O\left(\overline{A_{1}}, \ldots, \overline{A_{n}}\right)$ are nested and the extremes of $\underline{\bar{O}}\left(A_{1}, \ldots, A_{n}\right)$ coincide with their respective minimum and maximum (with respect to the partial ordering determined by Zadeh's inclusion):

Proposition 5: Let us consider the partial ordered-set $(\mathcal{F}(U), \subseteq)$. Let $O: \mathcal{F}(U) \times \stackrel{(n)}{.} \times \mathcal{F}(U) \rightarrow \mathcal{F}(U)$ be either increasing in all the components or decreasing in all of them. The extremes of the max-min extension of $O: \mathcal{F}(U) \times(n)$. $\times \mathcal{F}(U) \rightarrow \mathcal{F}(U), \underline{\bar{O}}=[\underline{O}, \bar{O}]$ can be alternatively expressed as:

$$
\begin{aligned}
& \underline{O}\left(A_{1}, \ldots, A_{n}\right)=\min _{\subseteq}\left\{O\left(\underline{A_{1}}, \ldots, \underline{A_{n}}\right), O\left(\overline{A_{1}}, \ldots, \overline{A_{n}}\right)\right\} \\
& \bar{O}\left(A_{1}, \ldots, A_{n}\right)=\max _{\subseteq}\left\{O\left(\underline{A_{1}}, \ldots, \underline{A_{n}}\right), O\left(\overline{A_{1}}, \ldots, \overline{A_{n}}\right)\right\} .
\end{aligned}
$$

The following result is a consequence of Proposition 5 and Corollary 1, and it relates Definitions 1 and 8:

Corollary 2: Consider the partial ordered-set $(\mathcal{F}(U), \subseteq)$ and an operator $O: \mathcal{F}(U) \times \stackrel{(n)}{.} \times \mathcal{F}(U) \rightarrow \mathcal{F}(U)$.

- If $[O]\left(A_{1}, \ldots, A_{n}\right)$ has a minimum $[O]\left(A_{1}, \ldots, A_{n}\right)$ and a maximum $\overline{[O]}\left(A_{1}, \ldots, A_{n}\right)$ then:

$$
\begin{aligned}
& -\underline{[O]}\left(A_{1}, \ldots, A_{n}\right) \subseteq \underline{O}\left(A_{1}, \ldots A_{n}\right) . \\
& -\overline{\overline{[O]}}\left(A_{1}, \ldots, A_{n}\right) \supseteq \bar{O}\left(A_{1}, \ldots A_{n}\right) .
\end{aligned}
$$

- If $O$ is either increasing in all the components or decreasing in all of them, then:

$\underline{\underline{[O]}}\left(A_{1}, \ldots, A_{n}\right) \quad=\quad \underline{O}\left(A_{1}, \ldots A_{n}\right) \quad$ and $\left.\overline{\overline{[O]}}\left(A_{1}, \ldots, A_{n}\right)=\bar{O}\left(A_{1}, \ldots A_{n}\right)\right\}$.

- If there exists a continuous mapping $s:[0,1] \times \ldots \times$ $[0,1] \rightarrow[0,1]$ such that $O: \mathcal{F}(U) \times \ldots \times \mathcal{F}(U) \rightarrow \mathcal{F}(U)$ is defined from $s$ as follows:

$$
O\left(X_{1}, \ldots, X_{n}\right)(x)=s\left(X_{1}(x), \ldots, X_{n}(x)\right), \forall x \in U,
$$

where $s$ is either increasing in all the components or decreasing in all of them, then $[O]=\bar{O}$.

The implications of this result are twofold. Firstly, we see that the most common extended operators (union, intersection, complement, contraction, dilation) do not only follow the general formulation proposed in Definition 1, but also the one considered in Definition 8 and they can also be expressed as particular cases of Equation 10. Notice that each of the aforementioned operators is generated either by a continuous mapping of the form $s:[0,1] \times[0,1] \rightarrow[0,1]$ (binary operators) or of the form $s^{\prime}:[0,1] \rightarrow[0,1]$ (unary operators) as follows:

$$
\begin{gathered}
O\left(A_{1}, A_{2}\right)(x)=s\left(A_{1}(x), A_{2}(x)\right), \forall x \in U . \\
O(A)(x)=s^{\prime}(A(x)), \forall x \in U .
\end{gathered}
$$

Secondly, this alternative formulation of Definition 8 allows us to find some formal connections between the extended operators analyzed here and some extensions of real-valued functions considered in the literature and studied in the next subsection.

2) Extensions of real-valued mappings: In this subsection we will review some extensions of real-valued mappings whose images can be written in terms of the images of the extremes of the corresponding IVFSs, according to a formulation similar to the one considered in Equation 10. The degree of compatibility between IVF sets of Gorzalczany [20] can be cast into this category. Furthermore, other previously mentioned notions such as the probability of A-IFS events of Riecan [28] or the cardinality of an IVF set [26] can not only be regarded as instances of the general formulation provided in Definition 2, but also as examples of Definition 9 given below, as a consequence of Proposition 4. All these extended definitions deal with completely different kinds of notions, but if we analyze them from a purely formal point of view, we can find clear connections. It is out of the scope of this paper to review an exhaustive list of definitions that follow the same general formulation. The interested reader will easily find formal connections with other extended definitions that follow a similar pattern.

Let us start by proposing the general max-min extension of a real-valued mapping $f: \mathcal{F}(U) \times(n) \times \mathcal{F}(U) \rightarrow \mathbb{R}$ :

Definition 9: The max-min extension $\bar{f}=[\underline{f}, \bar{f}]$ : $I V F S(U) \times \stackrel{(n)}{I} \times \operatorname{IVFS}(U) \rightarrow \mathbb{R}$ of a mapping $f:$ $\mathcal{F}(U) \times \stackrel{(n)}{ }) \times \mathcal{F}(U) \rightarrow \mathbb{R}$ is defined as follows:

$$
\begin{aligned}
\underline{f}\left(A_{1}, \ldots, A_{n}\right) & =\min \left\{f\left(\underline{A_{1}}, \ldots, \underline{A_{n}}\right), f\left(\overline{A_{1}}, \ldots, \overline{A_{n}}\right)\right\} \\
\bar{f}\left(A_{1}, \ldots, A_{n}\right) & =\max \left\{f\left(\underline{A_{1}}, \ldots, \underline{A_{n}}\right), f\left(\overline{A_{1}}, \ldots, \overline{A_{n}}\right)\right\}
\end{aligned}
$$


This formulation reminds us to Equations 10. There, the minimum and the maximum where calculated with respect to the partial ordering determined by inclusion of fuzzy sets. We required the operator $O$ to be either increasing in all the components or decreasing in all of them, in order to guarantee the existence of the minimum and maximum of $O\left(\underline{A_{1}}, \ldots, \underline{A_{n}}\right)$ and $O\left(\overline{A_{1}}, \ldots, \overline{A_{n}}\right)$, with respect to this partial ordering. As a counterpart, the maximum and the minimum calculated in Equation 12 always exist, because $f$ is a real-valued mapping, and we consider the usual (total) ordering on $\mathbb{R}$. Thus, no requirement regarding the monotonicity of $f$ is needed in order to guarantee the existence of the right hand side term in Equation 12.

The notion of degree of compatibility between IVF introduced by Gorzalczany defined in [20] illustrates the above general definition:

Definition 10: The compatibility degree $\underline{\bar{\Gamma}}(A, B)$ of an IVF set $A=[\underline{A}, \bar{A}]$ such that there is at least one $x \in U$ with $\underline{A}(x)>0$ with an IVF set $B=[\underline{B}, \bar{B}]$ is the closed interval $\bar{\Gamma}(A, B)$ whose minimum and maximum values are respectively:

$$
\min \left\{\frac{\max _{x \in U}(\underline{A} \cap \underline{B})(x)}{\max _{x \in U} \underline{A}(x)}, \frac{\max _{x \in U}(\bar{A} \cap \bar{B})(x)}{\max _{x \in U} \bar{A}(x)}\right\}
$$

and

$$
\max \left\{\frac{\max _{x \in U}(\underline{A} \cap \underline{B})(x)}{\max _{x \in U} \underline{A}(x)}, \frac{\max _{x \in U}(\bar{A} \cap \bar{B})(x)}{\max _{x \in U} \bar{A}(x)}\right\} .
$$

If we restrict ourselves to the particular case where $A(x)=$ $\underline{A}(x)=\bar{A}(x)$ and $B(x)=\underline{B}(x)=\bar{B}(x)$ it comes down to the following compatibility index between fuzzy sets:

$$
\Gamma(A, B)=\frac{\max _{x \in U}(A \cap B)(x)}{\max _{x \in U} A(x)} .
$$

We clearly observe for every $A, B \in I V F S(U)$, that

$\underline{\bar{\Gamma}}(A, B)=[\min \{\Gamma(\underline{A}, \underline{B}), \Gamma(\bar{A}, \bar{B})\}, \max \{\Gamma(\underline{A}, \underline{B}), \Gamma(\bar{A}, \bar{B})\}]$

As we have mentioned above, the probability of A-IF events ( [22], [28], [32]), the cardinality of A-IFSs ( [8], [33]) and of IVFSs ( [34]), among others, not only follow the general formulation provided in Definition 2 but also the one given in Definition 9. These two general formulations do not coincide in general, but they do under some particular conditions. The following result links both of them:

Proposition 6: Let us consider a mapping $f: \mathcal{F}(U) \times \stackrel{(n)}{\text {. }}$. $\times \mathcal{F}(U) \rightarrow \mathbb{R}$. Let $\underline{\bar{f}}=[\underline{f}, \bar{f}]$ and $[f]$ respectively denote the max-min and the set-valued arithmetic extensions. Then:

$$
\begin{aligned}
& \underline{[f]}\left(A_{1}, \stackrel{(n)}{.}, A_{n}\right) \leq \underline{f}\left(A_{1}, \stackrel{(n)}{.}, A_{n}\right), \\
& \overline{[f]}\left(A_{1}, \stackrel{(n)}{.}, A_{n}\right) \geq \bar{f}\left(A_{1}, \stackrel{(n)}{.}, A_{n}\right), \\
& \forall\left(A_{1}, \stackrel{(n)}{.}, A_{n}\right) \in I V F(U) \times \stackrel{(n)}{.} \times I V F(U) .
\end{aligned}
$$

Furthermore, the equality holds in both equations (Eq. 13 and 14) if $f$ is monotone (increasing or decreasing) in all the arguments with respect to Zadeh's fuzzy set inclusion.

Proof: The first part is due to the fact that the collection $\left\{\left(X_{1}, \ldots, X_{n}\right): \underline{A}_{i} \subseteq X \subseteq \bar{A}_{i}, i=1, \ldots, n\right\}$ includes the set $\left\{\left(\underline{A}_{1}, \ldots, \underline{A}_{n}\right),\left(\bar{A}_{1}, \ldots, \bar{A}_{n}\right)\right\}$. The second part is a consequence of the first part of Proposition 4.

According to the last result, we easily observe that the probability of A-IF events ( [22], [28], [32]) and the cardinality defined in [26] do not also match Definition 9 but also Definition 2, since both Zadeh's probability and the cardinality of fuzzy sets are monotone increasing functions wrt fuzzy set inclusion. However, any inclusion index on $\mathcal{F}(U) \times \mathcal{F}(U)$ satisfying the five axioms of Cornelis et al. [15] is decreasing wrt the first component and increasing wrt the second one, and this is the reason why its max-min inclusion (the extension via Equation 12) is included, but does not necessarily coincide with (the convex hull of) its set-valued inclusion.

\section{Third category: The max-min-varied extension}

Some extended definitions do not match Equations 10 and 12 but are somehow related to them. The degree of inclusion between A-IFSs of Bustince [9] is one of them. In this subsection we will provide another general formulation that can be seen as a variant of Equations 10 and 12 and encompasses some of those definitions. At the end of the subsection, we will provide a result relating the properties of the initial mapping $f$ and the extended mapping $\left[f_{L}, f_{U}\right]$ constructed in accordance with the new alternative equation.

Let us start by discussing the notion of degree of inclusion between A-IFSs introduced by Bustince in [9] which generalizes the "inclusion degree" between fuzzy sets defined by Sinha and Dougherty [30]:

Definition 11: Let us consider a continuous and increasing bijection $g:[0,1] \rightarrow[0,1]$. Given two fuzzy subsets $A, B \in$ $\mathcal{F}(U)$, the degree of inclusion of $A \in \mathcal{F}(U)$ wrt $B \in \mathcal{F}(U)$ is defined as:

$$
\Upsilon(A, B)=\inf _{x \in U} \min \{1,1-g(A(x))+g(B(x))\} .
$$

If $\gamma:[0,1] \times[0,1] \rightarrow[0,1]$ is defined as $\gamma(a, b)=$ $\min \{1,1-g(a)+g(b)\}, \forall a, b \in[0,1]$, we can write

$$
\Upsilon(A, B)=\inf _{x \in U} \gamma(A(x), B(x)) .
$$

Then, Bustince's generalization of Definition 11 for IVFSs can be expressed as:

Definition 12: Given two IVFSs, $A=[\underline{A}, \bar{A}], B=[\underline{B}, \bar{B}] \in$ IVFS $(U)$, with $U$ finite, the interval-valued inclusion grade of $(A, B)$ is the closed interval $\Upsilon(A, B)$ with respective bounds:

$$
\begin{aligned}
& \Upsilon_{L}(A, B)=\inf _{x \in U} \min \{\gamma(\underline{A}(x), \underline{B}(x)), \gamma(\bar{A}(x), \bar{B}(x))\}, \\
& \Upsilon_{R}(A, B)=\inf _{x \in U} \max \{\gamma(\underline{A}(x), \underline{B}(x)), \gamma(\bar{A}(x), \bar{B}(x))\} .
\end{aligned}
$$

Taking into account that the infimum is in fact a minimum and it is increasing in all the components, we can write:

$$
\Upsilon_{L}(A, B)=\min _{(X, Y) \in \mathcal{F}_{2}(A, B)} \Upsilon(X, Y)
$$

and

$$
\Upsilon_{R}(A, B)=\max _{(X, Y) \in \mathcal{F}_{2}(A, B)} \Upsilon(X, Y)
$$


where $\mathcal{F}_{2}(A, B)=\{(X, Y) \in \mathcal{F}(U) \times \mathcal{F}(U)$ $(X(x), Y(x)) \in\{(\underline{A}(x), \underline{B}(x)),(\bar{A}(x), \bar{B}(x))\}$.

This formulation is a particular instance of a third general formulation provided in Definition 13.

Definition 13: The max-min-varied extension of $f: \mathcal{F}(U) \times$ $\ldots \times \mathcal{F}(U) \rightarrow \mathbb{R}$ is defined as $\left[f_{l}, f_{R}\right]: I V F S(U) \times$ $\ldots I V F S(U) \rightarrow \mathbb{R}$ as follows:

$$
\begin{aligned}
& f_{L}\left(A_{1}, \ldots, A_{n}\right)=\min _{\left(X_{1}, \ldots, X_{n}\right) \in \mathcal{F}_{2}\left(A_{1}, \ldots, A_{n}\right)} f\left(X_{1}, \ldots, X_{n}\right), \\
& f_{R}\left(A_{1}, \ldots, A_{n}\right)=\max _{\left(X_{1}, \ldots, X_{n}\right) \in \mathcal{F}_{2}\left(A_{1}, \ldots, A_{n}\right)} f\left(X_{1}, \ldots, X_{n}\right),
\end{aligned}
$$

where

$$
\begin{array}{ccc}
\mathcal{F}_{2}\left(A_{1}, \ldots, A_{n}\right) & = & \left\{\left(X_{1}, \ldots, X_{n}\right)\right. \\
\left(X_{1}(x), \ldots X_{n}(x)\right) & = & \left(A_{1}(x), \ldots, \underline{A}_{n}(x)\right), \\
\left.\left(X_{1}(x), \ldots X_{n}(x)\right)=\left(\overline{A_{1}}(x), \ldots, \overline{A_{n}}(x)\right\}, \forall x \in U\right\} .
\end{array}
$$

A notion of interval-valued similarity measure given in [9] can be also regarded as a particular case of Definition 13 .

\section{Formal relations between the three set-valued extensions}

The following result relates the three general formulae introduced in Definitions 2, 9 and 13:

Proposition 7:

$$
\begin{aligned}
& \underline{[f]}\left(A_{1}, \ldots, A_{n}\right) \leq f_{L}\left(A_{1}, \ldots, A_{n}\right) \leq \underline{f}\left(A_{1}, \ldots, A_{n}\right) \\
& \overline{[f]}\left(A_{1}, \ldots, A_{n}\right) \geq f_{R}\left(A_{1}, \ldots, A_{n}\right) \leq \bar{f}\left(A_{1}, \ldots, A_{n}\right)
\end{aligned}
$$

Proof: Given a tuple of IVFSs $\left(A_{1}, \ldots, A_{n}\right)$, consider the following three subsets of $\mathcal{F}(U) \times \ldots \times \mathcal{F}(U)$ (collections of tuples of fuzzy subsets of $U)$ :

- $\mathcal{F}_{1}\left(A_{1}, \ldots, A_{n}\right)=\left\{\left(X_{1}, \ldots, X_{n}\right): \underline{A_{i}} \subseteq X_{i} \subseteq \overline{A_{i}}, i=\right.$ $1, \ldots, n\}$.

- $\mathcal{F}_{2}\left(A_{1}, \ldots, A_{n}\right) \quad=\left\{\left(X_{1}, \ldots, X_{n}\right) \quad\right.$ : $\left(X_{1}(x), \ldots X_{n}(x)\right)=\left(\underline{A_{1}}(x), \ldots, \underline{A}_{n}(x)\right), \quad$ or $\left.\left(X_{1}(x), \ldots X_{n}(x)\right)=\left(\overline{A_{1}}(x), \ldots, \overline{A_{n}}(x)\right\}, \forall x \in U\right\}$.

- $\mathcal{F}_{3}\left(A_{1}, \ldots, A_{n}\right)=\left\{\left(\underline{A_{1}}, \ldots, \underline{A_{n}}\right),\left(\underline{A_{1}}, \ldots, \underline{A_{n}}\right)\right\}$.

We will shortly denote them as $\mathcal{F}_{1}, \mathcal{F}_{2}$ and $\mathcal{F}_{3}$, respectively. The three extensions can be expressed in terms of those collections of tuples as follows:

- $[f]\left(A_{1}, \ldots, A_{n}\right)=\inf _{\left(X_{1}, \ldots, X_{n}\right) \in \mathcal{F}_{1}} f\left(X_{1}, \ldots, X_{n}\right)$, and $\overline{\overline{[f]}}\left(A_{1}, \ldots, A_{n}\right)=\sup _{\left(X_{1}, \ldots, X_{n}\right) \in \mathcal{F}_{1}} f\left(X_{1}, \ldots, X_{n}\right)$,

- $f_{L}\left(A_{1}, \ldots, A_{n}\right)=\min _{\left(X_{1}, \ldots, X_{n}\right) \in \mathcal{F}_{2}} f\left(X_{1}, \ldots, X_{n}\right)$ and $f_{R}\left(A_{1}, \ldots, A_{n}\right)$ $\max _{\left(X_{1}, \ldots, X_{n}\right) \in \mathcal{F}_{2}} f\left(X_{1}, \ldots, X_{n}\right)$.

- $\underline{\underline{f}}\left(A_{1}, \ldots, A_{n}\right)=\min _{\left(X_{1}, \ldots, X_{n}\right) \in \mathcal{F}_{3}} f\left(X_{1}, \ldots, X_{n}\right)$ and $\overline{\bar{f}}\left(A_{1}, \ldots, A_{n}\right)=$ $\max _{\left(X_{1}, \ldots, X_{n}\right) \in \mathcal{F}_{3}} f\left(X_{1}, \ldots, X_{n}\right)$.

Clearly, $\mathcal{F}_{1} \supseteq \mathcal{F}_{2} \supseteq \mathcal{F}_{3}$ and therefore, according to the properties of the minimum and the maximum, the above inequalities hold.

Summarizing, the set-valued extension of $f$ gives birth to a set-valued mapping whose images are regarded as the collection of "feasible images" through $f$, according to the incomplete information provided about the "truth" tuple of fuzzy sets, determined by means of a tuple of IVFSs. The other two alternative extensions do not match such a "conjunctive" or "epistemic" ( [17]) interpretation. Notwithstanding, they are easier to compute in general, and this is perhaps one of the reasons why they have been considered in the literature. According to Proposition 7, their images are always included in those of the set-valued extension. Finding appropriate interpretations for both of them remains an open problem. Let us illustrate the differences between the three extensions with a couple of examples:

Example 2: Let us consider the following axiomatic definition of similarity between fuzzy sets. It particularizes the definition originally given by Dengfeng and Chuntian in [18] in the general context of A-IFSs. (Different variants of this notion can be found in the literature. A formal relational study is provided in [16]).

$S: \mathcal{F}(U) \times \mathcal{F}(U) \rightarrow \mathbb{R}$ is a similarity measure if it satisfies the following properties:

(Sim 1) $0 \leq S(A, B) \leq 1, \forall A, B \in \mathcal{F}(U)$.

(Sim 2) $A=B \Rightarrow S(A, B)=1$.

(Sim 3) $S(A, B)=S(B, A), \forall A, B \in \mathcal{F}(U)$.

(Sim 4) If $A \subseteq B \subseteq C$ then $S(A, C) \leq S(A, B)$.

(Sim 5) If $A \subseteq B \subseteq C$ then $S(A, C) \leq S(B, C)$.

Let $s$ be a similarity measure, and let $[S] \leq S_{L} \leq \underline{S}$ and $\overline{[S]} \geq S_{R} \geq \bar{S}$ denote the lower and upper bounds of the three extensions considered in this section. Let us see how the similarity between an IVFS $A=[\underline{A}, \bar{A}] \in I V F S(U)$ and itself is computed according to each of the three definitions:

- The extreme values of the set-valued extension are respectively:

$$
\begin{aligned}
& \text { - } \underline{[S]}(A, A)=\inf _{(X, Y): \underline{A} \subseteq X \subseteq \bar{A}, \underline{A} \subseteq Y \subseteq \bar{A}} S(X, Y)= \\
& \overline{s(\underline{A}}, \bar{A}) \text { and } \\
& \text { - } \overline{[S]}(A, A)=\sup _{(X, Y): \underline{A} \subseteq X \subseteq \bar{A}, \underline{A} \subseteq Y \subseteq \bar{A}} S(X, Y)=
\end{aligned}
$$

The set $[S](A, A)$ represents in fact, the collection of possible values for the similarity between two fuzzy sets $X$ and $Y$, when our incomplete information about each of them is represented by means of the IVFS $[\underline{A}, \bar{A}]$. We know that the degree of similarity between $X$ and $Y$ is greater than or equal to $S(\underline{A}, \bar{A})$.

- $[\underline{S}(A, A), \bar{S}(A, A)]=\{1\}$.

The max-min extension $[\underline{S}(A, A), \bar{S}(A, A)]$ seems to represent the similarity between $A$ and itself, from a "disjunctive" or "ontic" point of view ( [17]) by means of an interval. Under this interpretation, the interval-valued set is regarded as an "object" or an "element" in the collection $\operatorname{IVFS}(U)$ and not as a piece of incomplete information about an ill-known fuzzy set.

- $\left[S_{L}(A, A), S_{R}(A, A)\right]=\{1\}$.

The max-min varied extension coincides, in this example, with the max-min extension, but they do not coincide in general, as we illustrate in the next example. It compares the three extensions with an example about the notion of inclusion index.

\section{Example 3:}

Let us consider Bustince's fuzzy sets inclusion index $\Upsilon$ : $\mathcal{F}(U) \times \mathcal{F}(U) \rightarrow[0,1]$ recalled in Definition 11:

$$
\Upsilon(A, B)=\inf _{x \in U} \gamma(A(x), B(x)),
$$


where $\gamma(a, b)=\min \{1,1-g(a)+g(b)\}$, with $g:[0,1] \rightarrow$ $[0,1]$ increasing and $g(0)=0$, and $g(1)=1$. Let us consider the three extensions of $\Upsilon$ respectively based on Definitions 2, 12 and 13. They would respectively formulated as follows:

- Set-valued extension: Given two IVF sets $A, B$, their setvalued inclusion index is:

$$
[\Upsilon](A, B)=\{\Upsilon(X, Y): \underline{A} \subseteq X \subseteq \bar{A}\} .
$$

The extremes of the set-valued extension correspond to Grzegorzewski's proposal, in the sense that

$$
[\Upsilon](A, B)=\Upsilon(\bar{A}, \underline{B})=N I(A, B),
$$

and

$$
\overline{[\Upsilon]}(A, B)=\Upsilon(\underline{A}, \bar{B})=P I(A, B) .
$$

As we have previously recalled, Grzegorzewski does not consider the specific index $\Upsilon$, but a general family of fuzzy-set inclusion indices satisfying the five axioms of Cornelis et al. As we have clarified in Subsection III-A2, when such an inclusion index is generated by means of a continuous implication function (as it happens with the mapping $\gamma(x, y)=\min \{1,1-g(x)+g(y)\})$, the images of its set-valued extension are intervals of the form $[N I(A, B), P I(A, B)]$.

- Max-min extension: The max-min inclusion index of $(A, B)$ is defined as the interval:

$$
\underline{\Upsilon}(A, B)=[\underline{\Upsilon}(A, B), \bar{\Upsilon}(A, B)],
$$

where $\Upsilon(A, B)=\min \{\Upsilon(\underline{A}, \underline{B}), \Upsilon(\bar{A}, \bar{B})\}$ and $\bar{\Upsilon}(A, B)=\max \{\Upsilon(\underline{A}, \underline{B}), \Upsilon(\bar{A}, \bar{B})\}$. Up to our knowledge, the max-min extension of $\Upsilon$ has not been previously considered in the literature.

- Max-min-varied extension:

$$
\Upsilon_{(L, R)}(A, B)=\left[\Upsilon_{L}(A, B), \Upsilon_{R}(A, B)\right],
$$

with $\Upsilon_{L}(A, B)=\min _{(X, Y) \in \mathcal{F}_{2}} \Upsilon(X, Y)$, and $\Upsilon_{R}(A, B)=\max _{(X, Y) \in \mathcal{F}_{2}} \Upsilon(X, Y)$. The max-min varied extension coincides with the formulation proposed by Bustince.

Next, we will illustrate some differences between the three above extensions of $\Upsilon$. Take a universe $U=\left\{x_{1}, x_{2}\right\}$ and the IVFSs $A=\left\{\left(x_{1},[0.1,0.5]\right),\left(x_{2},[0.3,0.3]\right)\right\}$ and $B=\left\{\left(x_{1},[0.2,0.4]\right),\left(x_{2},[0.2,0.4]\right)\right\}$. Then:

- For the set-valued extension, we have, according to Grzegorzewski's proposal, that $[\Upsilon](A, B)=[0.7,1]$ and this interval can be understood as representing our incomplete information about the value of the inclusion index associated to the "true" pair of fuzzy sets $(X, Y)$ from which all we know is that $\underline{A} \subseteq X \subseteq \bar{A}$ and $\underline{B} \subseteq Y \subseteq \bar{B}$.

- For the Max-min extension.- In this case, $\Upsilon(A, B)=$ $\{0.9\}$ and this index represents the degree of inclusion of $A=[\underline{A}, \bar{A}]$ with respect to $B=[\underline{B}, \bar{B}]$, both of them considered as objects, and not as imprecise representations of two ill-observed fuzzy sets. In fact, it could be understood as the degree of inclusion in relation with the lattice ordering between interval-valued membership functions.
- For the Max-min-varied extension, since

$$
\Upsilon(X, Y)=\inf _{x \in U} \gamma(X(x), Y(x)), \forall(X, Y) \in \mathcal{F}(U) \times \mathcal{F}(U),
$$

it can be proved that the two lower bounds $\Upsilon$ and $\Upsilon_{L}$ do coincide, so $\Upsilon_{L}(A, B)=0.9$. However, $\Upsilon_{R}(A, B)$ is equal to 1 . In fact, there exists a pair of fuzzy sets $(X, Y) \in \mathcal{F}_{2}$ such that $\Upsilon(X, Y)=1$. It is the pair of fuzzy sets $\left(X\left(x_{1}\right), Y\left(x_{1}\right)\right)=(0.1,0.2)=$ $\left(\underline{A}\left(x_{1}\right), \underline{B}\left(x_{1}\right)\right)$ and $\left(X\left(x_{2}\right), Y\left(x_{2}\right)\right)=(0.3,0.4)=$ $\left(\bar{A}\left(x_{2}\right), \bar{B}\left(x_{2}\right)\right)$. Such a pair of fuzzy sets does not belong to $\mathcal{F}_{3}=\{(\underline{A}, \underline{B}),(\bar{A}, \bar{B})\}$. As we observe, the maxmin varied extension of $\Upsilon$ does not coincide in general with the max-min extension (calculated in the previous paragraph for this example). Further studies about the convenience of each of them in each particular problem of application would be of interest, but fall out of the scope of this general paper.

\section{CONCLUding REMARKS}

In this work, we have presented a categorization of many extensions of operators from the fuzzy setting to the IVFS or A-IFS settings. This categorization may help to simplify some proofs about their properties, as well as about the properties of possible future definitions. But it is also useful from a semantic point of view. As we have observed, the same FS notion admits at least three natural ways of extending it: the setvalued extension, that matches the conjunctive interpretation of sets, the max-min extension, that seems to match an ontic interpretation, and the max-min-varied extension, that offers a kind of compromise between both. These three views have been illustrated with some examples about the inclusion index and the degree of similarity. Let the reader notice that we have focused on set-valued extensions of different FS notions. A second part of our work will deal with point-valued extensions and their formal relations with the ones considered here. It would be natural to go one step beyond, and deal with even more generalized versions of those definitions in an encompassing lattice framework. We intend to develop such an study in future works. Finally, different approaches to the notion of extension of a fuzzy set, as the one of intuitionistic fuzzy multidimensional set discussed in the introduction, will also be considered in future researches, looking for a link (which is not obvious) among the different resulting algebraical structures.

\section{ACKNOWLEDGEMENTS}

This work is partially supported by TIN2014-56967-R and TIN2017-84804-R (Spanish Ministry of Science and Innovation), TIN2016-77356-P(AEI/FEDER, UE) and FC-15GRUPIN14-073 (Regional Ministry of the Principality of Asturias).

\section{REFERENCES}

[1] M. Asiain, H. Bustince, B. Bedregal, G. P. Dimuro, Z. Takac, M Baczynski, H. Zapata, Interval-valued implications and interval-valued similarity measures with admissible orders, submitted.

[2] K. Atanassov, Intuitionistic fuzzy sets, Fuzzy Sets and Systems 20 (1986) 87-96. 
[3] K. Atanassov, G. Gargov, Interval valued intuitionistic fuzzy sets, Fuzzy Sets and Systems 31 (1989) 343-349.

[4] K. Atanassov, E. Szmidt, J. Kacprzyk, On intuitionistic fuzzy multidimensional sets, Issues in Intuitionistic Fuzzy Sets and Generalized Nets 7 (2008) 1-6.

[5] K. Atanassov, E. Szmidt, J. Kacprzyk, P. Rangasamy. On intuitionistic fuzzy multi-dimensional sets. Part 2. Advances in Fuzzy Sets, Intuitionistic Fuzzy Sets, Generalized Nets and Related Topics. Vol. I: Foundations, Academic Publishing House EXIT, Warszawa, 2008, 4351.

[6] K. Atanassov, E. Szmidt, J. Kacprzyk, On intuitionistic fuzzy multidimensional sets. Part 3. Developments in Fuzzy Sets, Intuitionistic Fuzzy Sets, Generalized Nets and Related Topics, Vol. I: Foundations. Warsaw, SRI Polush Academy of Sciences, 2010, 19-26.

[7] K. Atanassov, K., E. Szmidt, J. Kacprzyk, On intuitionistic fuzzy multidimensional sets. Part 4. Notes on Intuitionistic Fuzzy Sets 17 (2) (2011) $1-7$.

[8] L. Atanassova, Remarks on the cardinality of the intuitionistic fuzzy sets, Fuzzy sets and Systems, 75 (1995), 399-400.

[9] H. Bustince, Indicator of inclusion grade for interval-valued fuzzy sets. Application to approximate reasoning based on interval-valued fuzzy sets. Int. J. Approx. Reason. 23(3) (2000) 137-209.

[10] H. Bustince, E. Barrenechea, M. Pagola, Generation of interval-valued fuzzy and atanassov's intuitionistic fuzzy connectives from fuzzy connectives and from Ka operators: Laws for conjunctions and disjunctions, amplitude, International Journal of Intelligent Systems 23 (6)(2008) 680714

[11] H. Bustince, V. Mohedano, E. Barrenechea, M. Pagola, Definition and con- struction of fuzzy DI-subsethood measures, Information Sciences 176 (21) (2006) 3190Ü3231.

[12] H. Bustince, E. Barrenechea, M. Pagola, J. Fernandez, Z.S. Xu, B. Bedregal, J. Montero, H. Hagras, F. Herrera, B. De Baets, A historical account of types of fuzzy sets and their relationships, IEEE Transactions on Fuzzy Systems 24 (1) (2016) 179-194.

[13] H. Bustince, M. Pagola, E. Barrenechea, J. Fernandez, P. Melo-Pinto, P. Couto, H. R. Tizhoosh, J. Montero, Ignorance functions. An application to the calculation of the threshold in prostate ultrasound images, Fuzzy Sets and Systems 161 (1) (2010) 20-36.

[14] H. Bustince, J. Fernandez, H. Hagras, F. Herrera, M. Pagola, E. Barrenechea, Interval type-2 fuzzy sets are generalization of interval-valued fuzzy sets: toward a wider view on their relationship, IEEE Transactions on Fuzzy Systems 23 (5) (2015) 1876-1882.

[15] C. Cornelis, C. Van der Donck, E. Kerre, Sinha-Dougherty approach to the fuzzification of set inclusion revisited, Fuzzy Sets and Systems 134 (2003) 283-295.

[16] I. Couso, L. Garrido, L. Sánchez, Similarity and dissimilarity measures between fuzzy sets: A formal relational study, Information Sciences 229 (2013) 122-141

[17] I. Couso, D. Dubois, Statistical reasoning with set-valued information: Ontic vs. epistemic views, International Journal of Approximate Reasoning 55 (2014) 1502-1518.

[18] L. Dengfeng, C. Chuntian, New similarity measures of intuitionistic fuzzy sets and application to pattern recognition, Pattern Recognition Letters 23 (2002) 221-225.

[19] G. Deschrijver, E.E. Kerre, On the relationship between some extensions of fuzzy set theory, Fuzzy Sets and Systems 133 (2003) 227- 235.

[20] Gorzalczany, M. B, A method of inference in approximate reasoning based on interval-valued fuzzy sets. Fuzzy sets and systems, 21(1987) $1-17$.

[21] I. Grattan-Guinness, Fuzzy membership mapped onto interval and manyvalued quantities, Zeitschrift für mathematische Logik und Grundladen der Mathematik 22 (1976) 149-160.

[22] P. Grzegorzewski, Conditional probability and independence of intuitionistic fuzzy events, NIFS 6 (2000) 7-14.

[23] P. Grzegorzewski, On possible and necessary inclusion of intuitionistic fuzzy sets, Information Sciences 181 (2011) 342-350.

[24] A. De Luca and S. Termini, A definition of non probabilistic entropy in the settings of fuzzy set theory, Information and Control, vol. 20, (1972), pp. 301-312.

[25] O. Nempont, J. Atif, I. Bloch, A constraint propagation approach to structural model based image segmentation and recognition, Information Sciences 246 (2013) 1-27.

[26] A. Niewiadomski, Cylindric extensions of interval-valued fuzzy sets in data linguistic summaries, J Ambient Intell Human Comput (2013) 4:369-376.
[27] A. Niewiadomski, J. Ochelska, P. S. Szczepaniak, Interval-valued linguistic summaries of databases, Control and Cybernetics 35 (2006) 415443.

[28] B. Riečan, A descriptive definition of the probability on intuitionistic fuzzy sets,

[29] R. Sambuc, Fonctions $\Phi$-floues. Application à l'aide au diagnostic en pathologie thyroidienne, Ph.D. Thesis, Université de Marseille, France, 1975.

[30] D. Sinha, E. R. Dougherty, Fuzzification of set inclusion: theory and applications, Fuzzy Sets and Systems 55 (1993) 15-42.

[31] A. Stachowiak and K. Dyczkowski, A Similarity Measure with Uncertainty for Incompletely Known Fuzzy Sets, Fuzz IEEE 2013.

[32] E. Szmidt, J. Kacprzyk, A Concept of a Probability of an Intuitionistic Fuzzy Event, 1999 IEEE International Fuzzy Systems Conference Proceedings, 1346-1349.

[33] E. Szmidt, J. Kacprzyk, Entropy for intuitionistic fuzzy sets, Fuzzy Sets and Systems 118 (2001) 467-477.

[34] D. Wu, J. Mendel, Vector Similarity Measure for Interval Type-2 Fuzzy Sets, Fuzz IEEE 2007.

[35] M. Wygralak, Cardinalities of Fuzzy Sets, Springer, Berlin, Heidelberg, New York, 2003.

[36] Zadeh, L.A. The concept of a linguistic variable and its application to approximate reasoning-1, Information Sciences, (1975) 199-249.

[37] L.A. Zadeh, Probability measures of fuzzy events, Journal of Mathematical Analysis and Applications 23 (1968) 421-427.

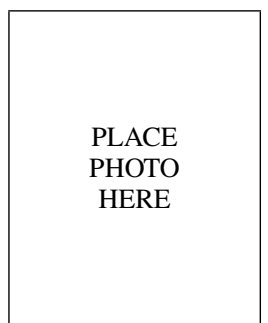

Inés Couso received the Ph.D. degree in Mathematics in 1999. Member of the Department of Statistics and O.R., University of Oviedo. She was an invited researcher at Université Paul Sabatier (Toulouse) (IRIT, 2009 and CIMI, 2015) and at Université de Montpellier 2 (LIRMM, 2011 and 2016). She currently serves as Area Editor for "Fuzzy Sets and Systems" and the "International Journal of Approximate Reasoning". Her research interests include foundations of fuzzy sets, imprecise probabilities, random sets, fuzzy random variables, statistics with coarse data and information theory.

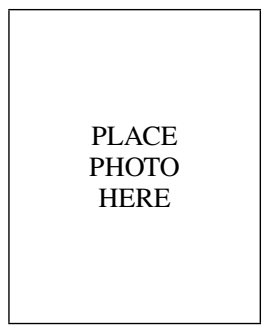

Humberto Bustince (M'08-SM'15) received the Ph.D. degree in Mathematics from the Public University of Navarra, Pamplona, Spain, in 1994. He is currently a Full Professor with the Department of Automatics and Computation of this University. $\mathrm{He}$ is the author of more than 200 published original articles. His research interests include fuzzy logic theory, extensions of fuzzy sets, fuzzy measures, aggregation functions, and fuzzy techniques for image processing. Dr. Bustince is an Editorial Board Member of IEEE Transactions on Fuzzy Systems, Fuzzy Sets and Systems and Information Fusion. 